Research Article

\title{
Finite-Time Stability and Stabilization of Switched Linear Time-Varying Systems with Time-Varying Delay
}

\author{
Lulu Feng $\mathbb{D}$ and Ping Zhao \\ School of Information Science and Engineering, Shandong Normal University, Jinan 250358, China \\ Correspondence should be addressed to Ping Zhao; zhaoping@amss.ac.cn
}

Received 27 September 2020; Revised 9 January 2021; Accepted 12 January 2021; Published 25 January 2021

Academic Editor: Delfim Soares Jr.

Copyright ( $\odot 2021$ Lulu Feng and Ping Zhao. This is an open access article distributed under the Creative Commons Attribution License, which permits unrestricted use, distribution, and reproduction in any medium, provided the original work is properly cited.

\begin{abstract}
This paper deals with the finite-time stability (FTS) of switched linear time-varying (SLTV) systems with time-varying delay. Firstly, based on Lyapunov-Krasovskii functional technique and average dwell time (ADT) approach, a sufficient criterion on FTS for SLTV systems with time-varying delay is obtained. For the SLTV system with delay and control input, based on the criterion, a state feedback controller is designed such that the closed-loop system is finite-time stable (FTS). Finally, an example is employed to illustrate the validity of our results.
\end{abstract}

\section{Introduction}

Switched systems are a special kind of hybrid dynamical systems, which consist of several subsystems and a switching law that orchestrates the switching between the subsystems [1]. It has been widely applied in intelligent traffic control [2], formation flying [3], sensor networks [4, 5], robotics [6, 7], and so on. In practice, time delays widely exist in many switched systems, which may cause some unexpected errors or even lead to crash; therefore, stability analysis for switched systems with time delay is particularly important and has been extensively studied. Most of the existing results focus on Lyapunov asymptotic stability (LAS). As we all know, LAS is defined over infinite-time interval. However, due to large state amplitude in the transient process, some asymptotic stable systems may be useless. Thus, it is more meaningful to study the stability in a fixed time interval. The concept of finite-time stability (FTS) was first introduced in 1960s [8,9]. A system is said to be finitetime stable (FTS) if its state does not exceed a certain bound during a specified time interval for a given bound on the initial condition. For such stability, there are many practical applications such as networked control systems [10] and network congestion control [11].

Up to now, many important results on FTS have been reported [12-15]. For instance, Ren et al. proposed a suitable event-driven communication scheme for the networked switched systems, and finite-time boundedness and inputoutput FTS are simultaneously considered [16]. Yu et al. investigated FTS of switched positive linear systems with time-varying delays using the ADT method, and a sufficient condition on FTS is provided for the case of time-invariant systems [17].

We can see that the majority of the existing literatures related to finite-time stability analysis are mainly on timeinvariant systems. However, many systems in practical applications are time-varying. For the active suspension system in auto diving, the vehicle sprung and unsprung masses vary with the loading conditions. To increase the control accuracy, this system should be represented by a time-varying system [18]. Therefore, the stability analysis for time-varying systems is very important and necessary. In the past few decades, the stability theory of linear time-invariant systems has developed considerably $[19,20]$, while that of linear time-varying (LTV) systems is comparatively slow. This is because the analysis of LTV systems is much more complicated than that of linear time-invariant systems. The state transition matrixes of LTV systems, which are generally needed to ascertain the properties of stability, are impossible to be derived in addition to particular cases [21-23]. So, we must find another way to analyze the stability of SLTV 
systems with time-varying delays. With the help of Lyapunov functions, a series of differential Lyapunov-inequality-based sufficient conditions is derived for FTS analysis. And, as is well known, general Lyapunov-Krasovskii functionals with more information on time delay are helpful for reducing conservatism $[24,25]$. Therefore, in this paper, we analyze FTS of SLTV systems with time-varying delays using the Lyapunov-Krasovskii functional method.

In this paper, the main contributions of this paper can be summarized as follows: (1) sufficient conditions on FTS of the switched system will be given under the case of LTV systems; (2) a state feedback controller will be designed for the SLTV system with time-varying delays.

The paper is organized as follows. The problem to be considered is formulated in Section 2. In Section 3, FTS criterions are derived in terms of linear matrix inequalities (LMIs). In Section 4, a state feedback controller will be designed which can guarantee the FTS of the systems. In Section 5, a numerical example will be given to show the effectiveness of the proposed method. Section 6 concludes the paper.

\section{Preliminaries}

$\mathbb{R}^{n}$ and $\mathbb{R}^{n \times m}$ denote, respectively, $n$-dimensional real space and $n \times m$-dimensional real matrix space. $\lambda_{\text {min }}(R)$ denotes the minimal of all eigenvalues of matrix $R$ and $\lambda_{\max }(R)$ denotes the maximum of all eigenvalues of matrix $R . R^{T}$ denotes the transpose of matrix $R$. $I$ denotes identity matrix with an appropriate dimension.

Consider the following SLTV systems:

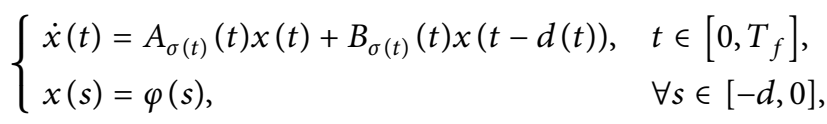

where $x(\cdot) \in \mathbb{R}^{n}$ is the system state and $T_{f}$ is a positive constant which represents the end time of system operation; switching signal $\sigma(t)$ is a piecewise constant function that maps from $\left[0, T_{f}\right]$ into the index set $M:=\{1,2, \ldots, m\}$; $A_{i}, B_{i}(i \in M)$ are system matrices with appropriate dimensions; $d(t)$ is the bounded time delay with $0 \leq d(t) \leq d$ and $\dot{d}(t) \leq \rho<1$ ( $d$ and $\rho$ are positive constants). The system state is denoted by $x\left(t, \sigma_{0}, \varphi\right)$ with initial mode $\sigma(0)=\sigma_{0}$ and initial state $\varphi(s), s \in[-d, 0]$. When clear from the context, we write $x(t)$ instead of $x\left(t, \sigma_{0}, \varphi\right)$.

For convenience, we shall introduce the following definitions.

Definition 1. System (1) is said to be FTS with respect to $\left(c_{1}, c_{2}, T_{f}\right)$ if

$$
\sup _{-d \leq s \leq 0} \varphi^{T}(s) \varphi(s) \leq c_{1} \Longrightarrow x^{T}(t) x(t) \leq c_{2}, \quad \forall t \in\left[0, T_{f}\right],
$$

where $c_{1}$ and $c_{2}$ are given positive constants with $c_{1} \leq c_{2}$.

Remark 1. Definition 1 implies that if system (1) is FTS, the state must be within the prescribed bound in the fixed time interval, and constants $c_{1}$ and $c_{2}$ are usually assigned according to the actual situation of the specific problem. In engineering applications, there are many systems that work on a finite-time interval, such as the climb process of hypersonic aircraft, and it must reach supersonic speed within the fixed time interval; in the process of spacecraft rendezvous and docking, the two spacecrafts must be combined in space orbit and structurally connected into a whole within the fixed time interval.

Definition 2. Let $N_{\sigma}(t, T)$ denote the switching number of $\sigma(t)$ over $[t, T), \forall T>t \geq 0$. If $N_{\sigma}(t, T) \leq N_{0}+\left(T-t / \tau_{D}\right)$ holds for some $\tau_{D}>0$ and $N_{0} \geq 0$, then $\tau_{D}$ and $N_{0}$ are called ADT and chattering bound, respectively.

\section{Finite-Time Stability Analysis}

In this section, using Lyapunov-Krasovkii functional technique and ADT approach, a sufficient condition on FTS of system (1) will be given as follows.

Theorem 1. System (1) is FTS with respect to $\left(c_{1}, c_{2}, T_{f}\right)$ if, for any $i \in M$, there are positive definite matrix-valued functions $P_{i}(t), Q_{i}(t), Z_{i}(t) \in \mathbb{R}^{n \times n}$, positive constant $\lambda$, and constant $\beta>1$, such that the following inequalities hold:

$$
\begin{gathered}
\left(\begin{array}{cc}
\Psi_{i}(t) & P_{i}(t) B_{i}(t) \\
B_{i}^{T}(t) P_{i}(t) & -e^{\lambda d} Q_{i}(t)(1-\rho)
\end{array}\right)<0, \\
P_{i}(t) \leq \beta P_{j}(t), Q_{i}(t) \leq \beta Q_{j}(t), Z_{i}(t) \leq \beta Z_{j}(t), \quad \forall i, j \in M, \\
\dot{Q}_{i}(t) \leq 0, \\
\dot{Z}_{i}(t) \leq 0,
\end{gathered}
$$

and the ADT satisfies

$$
\tau_{D}>\frac{T_{f} \ln \beta}{\ln c_{2}+\ln \lambda_{p}-\lambda T_{f}-\ln \eta_{1}+N_{0} \ln \beta},
$$

where

$$
\begin{aligned}
\Psi_{i}(t)= & A_{i}^{T}(t) P_{i}(t)+\dot{P}_{i}(t)+P_{i}(t) A_{i}(t)+Q_{i}(t) \\
& +d Z_{i}(t)-\lambda P_{i}(t), \\
\lambda_{p}= & \min _{i \in M}\left(\lambda_{\min }\left(P_{i}(t)\right)\right), \\
\eta_{1}= & \alpha_{1} c_{1}+d e^{\lambda d} \alpha_{2} c_{1}+d^{2} e^{\lambda d} \alpha_{3} c_{1}, \\
\alpha_{1}= & \max _{i \in M}\left\{\lambda_{\max }\left(P_{i}(0)\right)\right\}, \\
\alpha_{2}= & \max _{i \in M}\left\{\lambda_{\max }\left(Q_{i}(0)\right)\right\}, \\
\alpha_{3}= & \max _{i \in M}\left\{\lambda_{\max }\left(Z_{i}(0)\right)\right\} .
\end{aligned}
$$

Proof. Take the following Lyapunov-Krasovkii functional candidate: 


$$
V_{\sigma(t)}\left(t, x_{t}\right)=V_{\sigma(t), 1}\left(t, x_{t}\right)+V_{\sigma(t), 2}\left(t, x_{t}\right)+V_{\sigma(t), 3}\left(t, x_{t}\right),
$$

Calculating the derivatives of $V_{\sigma(t), 1}(t), V_{\sigma(t), 2}(t)$, and $V_{\sigma(t), 3}(t)$ with respect to $t$ along the trajectories of system (1), we can obtained

where

$$
\begin{aligned}
& V_{\sigma(t), 1}\left(t, x_{t}\right)=x^{T}(t) P_{\sigma(t)}(t) x(t) \\
& V_{\sigma(t), 2}\left(t, x_{t}\right)=\int_{t-d(t)}^{t} x^{T}(s) e^{\lambda(t-s)} Q_{\sigma(t)}(t) x(s) d s \\
& V_{\sigma(t), 3}\left(t, x_{t}\right)=\int_{-d}^{0} \int_{t+\theta}^{t} x^{T}(s) e^{\lambda(t-s)} Z_{\sigma(t)}(t) x(s) d s d \theta .
\end{aligned}
$$

$$
\begin{aligned}
\dot{V}_{\sigma(t), 1}\left(t, x_{t}\right)= & x^{T}(t)\left(A_{\sigma(t)}^{T}(t) P_{\sigma(t)}(t)+\dot{P}_{\sigma(t)}(t)+P_{\sigma(t)}(t) A_{\sigma(t)}(t)\right) x(t) \\
& +x^{T}(t-d(t)) B_{\sigma(t)}^{T}(t) P_{\sigma(t)}(t) x(t) \\
& +x_{\sigma(t)}^{T}(t) P_{\sigma(t)}(t) B_{\sigma(t)}(t) x(t-d(t)), \\
\dot{V}_{\sigma(t), 2}\left(t, x_{t}\right)= & \lambda V_{\sigma(t), 2}(t)+x^{T}(t) Q_{\sigma(t)}(t) x(t) \\
& -e^{\lambda d(t)} x^{T}(t-d(t)) Q_{\sigma(t)}(t) x(t-d(t))(1-\dot{d}(t)) \\
& +\int_{t-d(t)}^{t} e^{\lambda(t-s)} x^{T}(s) \dot{Q}_{\sigma(t)}(t) x(s) \mathrm{d} s, \\
\dot{V}_{\sigma(t), 3}\left(t, x_{t}\right)= & \lambda V_{\sigma(t), 3}(t)-\int_{t-d}^{t} e^{\lambda(t-s)} x^{T}(s) Z_{\sigma(t)}(t) x(s) d s \\
& +\int_{-d}^{0} \int_{t+\theta}^{t} e^{\lambda(t-s)} x^{T}(s) \dot{Z}_{\sigma(t)}(t) x(s) d s d \theta+d x^{T}(t) Z_{\sigma(t)}(t) x(t) .
\end{aligned}
$$

Furthermore,

$$
\begin{aligned}
& \dot{V}_{\sigma(t)}\left(t, x_{t}\right)-\lambda V_{\sigma(t)}\left(t, x_{t}\right) \\
& =x^{T}(t)\left(A_{\sigma(t)}^{T}(t) P_{\sigma(t)}(t)+\dot{P}_{\sigma(t)}(t)+P_{\sigma(t)}(t) A_{\sigma(t)}(t)+Q_{\sigma(t)}(t)+d Z_{\sigma(t)}(t)\right. \\
& \left.\quad-\lambda P_{\sigma(t)}(t)\right) x(t)+x^{T}(t-d(t))\left(B_{\sigma(t)}^{T}(t) P_{\sigma(t)}(t) x(t)\right. \\
& \left.\quad-e^{\lambda d(t)} Q_{\sigma(t)}(t) x(t-d(t))(1-\dot{d}(t))\right)+x^{T}(t) P_{\sigma(t)}(t) B_{\sigma(t)}(t) x(t-d(t)) \\
& \quad-\int_{t-d}^{t} e^{\lambda(t-s)} x^{T}(s) Z_{\sigma(t)}(t) x(s) \mathrm{d} s+\int_{t-d(t)}^{t} e^{\lambda(t-s)} x^{T}(s) \dot{Q}_{\sigma(t)}(t) x(s) d s \\
& \quad+\int_{-d}^{0} \int_{t+\theta}^{t} e^{\lambda(t-s)} x^{T}(s) \dot{Q}_{\sigma(t)}(t) x(s) d s d \theta \\
& \leq x^{T}(t)\left(A_{\sigma(t)}^{T}(t) P_{\sigma(t)}(t)+\dot{P}_{\sigma(t)}(t)+P_{\sigma(t)}(t) A_{\sigma(t)}(t)+Q_{\sigma(t)}(t)+d Z_{\sigma(t)}(t)\right. \\
& \left.\quad-\lambda P_{\sigma(t)}(t)\right) x(t)+x^{T}(t-d(t))\left(B_{\sigma(t)}^{T}(t) P_{\sigma(t)}(t) x(t)\right. \\
& \left.\quad-e^{\lambda d} Q_{\sigma(t)}(t) x(t-d(t))(1-\rho)\right)+x^{T}(t) P_{\sigma(t)}(t) B_{\sigma(t)}(t) x(t-d(t)) \\
& \quad-\int_{t-d}^{t} e^{\lambda(t-s)} x^{T}(s) Z_{\sigma(t)}(t) x(s) d s+\int_{t-d(t)}^{t} e^{\lambda(t-s)} x^{T}(s) \dot{Q}_{\sigma(t)}(t) x(s) d s \\
& \quad+\int_{-d}^{0} \int_{t+\theta}^{t} e^{\lambda(t-s)} x^{T}(s) \dot{Q}_{\sigma(t)}(t) x(s) d s d \theta .
\end{aligned}
$$


Inequality (12) can be rewritten as follows:

$$
\begin{aligned}
& \dot{V}_{\sigma(t)}\left(t, x_{t}\right)-\lambda V_{\sigma(t)}\left(t, x_{t}\right) \\
& \leq\left(\begin{array}{c}
x(t) \\
x(t-d(t))
\end{array}\right)^{T}\left(\begin{array}{cc}
\Psi_{\sigma(t)}(t) & P_{\sigma(t)}(t) B_{\sigma(t)}(t) \\
B_{\sigma(t)}^{T}(t) P_{\sigma(t)}(t) & -e^{\lambda d} Q_{\sigma(t)}(t)(1-\rho)
\end{array}\right)\left(\begin{array}{c}
x(t) \\
x(t-d(t))
\end{array}\right) \\
& \quad-\int_{t-d}^{t} x^{T}(s) e^{\lambda(t-s)} Z_{\sigma(t)}(t) x(s) d s+\int_{t-d}^{t} x^{T}(s) e^{\lambda(t-s)} \dot{Q}_{\sigma(t)}(t) x(s) d s \\
& \quad+\int_{-d}^{0} \int_{t+\theta}^{t} x^{T}(s) e^{\lambda(t-s)} \dot{Z}_{\sigma(t)}(t) x(s) d s d \theta .
\end{aligned}
$$

From conditions (3), (5), and (6), we can obtain

$$
\dot{V}_{\sigma(t)}\left(t, x_{t}\right)-\lambda V_{\sigma(t)}\left(t, x_{t}\right)<0 \text {. }
$$

Since $(d / \mathrm{d} t)\left(e^{-\lambda t} V_{i}\left(t, x_{t}\right)\right)=e^{-\lambda t}\left\{\dot{V}_{i}\left(t, x_{t}\right)-\lambda V_{i}\left(t, x_{t}\right)\right\}$, inequality (14) can be transformed into

$$
\frac{d}{d t}\left(e^{-\lambda t} V_{\sigma(t)}\left(t, x_{t}\right)\right)<0
$$

Let $t_{k}$ stand for the instant of $k$ th switching and $t_{k^{-}}$ denote the instant just before $t_{k}, \sigma(t)=\sigma\left(t_{k}\right), \forall t \in\left[t_{k}, t_{k+1}\right)$. Integrating both sides of (15) from $t_{k}$ to $t$, it follows that

$$
V_{\sigma\left(t_{k}\right)}\left(t, x_{t}\right) \leq e^{\lambda\left(t-t_{k}\right)} V_{\sigma\left(t_{k}\right)}\left(t_{k}, x_{t_{k}}\right), \quad t \in\left[t_{k}, t_{k+1}\right) .
$$

From (4) and the continuity of $x(t)$, the following inequalities hold:

$$
\begin{gathered}
V_{\sigma\left(t_{k}\right)}\left(t, x_{t}\right) \leq \beta e^{\lambda\left(t-t_{k}\right)} V_{\sigma\left(t_{k^{-}}\right)}\left(t_{k}, x_{t_{k}}\right), \\
V_{\sigma\left(t_{k^{-}}\right)}\left(t_{k}, x_{t_{k}}\right) \leq e^{\lambda\left(t_{k}-t_{k-1}\right)} V_{\sigma\left(t_{k-1}\right)}\left(t_{k-1}, x_{t_{k-1}}\right) .
\end{gathered}
$$

So,

$$
V_{\sigma(t)}\left(t, x_{t}\right) \leq \beta e^{\lambda\left(t-t_{k-1}\right)} V_{\sigma\left(t_{k-1}\right)}\left(t_{k-1}, x_{t_{k-1}}\right) .
$$

Iteratively, we can obtain

$$
V_{\sigma(t)}\left(t, x_{t}\right) \leq \beta^{N_{\sigma}(0, t)} e^{\lambda t} V_{\sigma_{0}}\left(0, x_{0}\right) .
$$

It is obvious that $V_{\sigma_{0}}\left(0, x_{0}\right)$ can be written as follows:

$$
\begin{aligned}
V_{\sigma_{0}}\left(0, x_{0}\right)= & x^{T}(0) P_{\sigma_{0}}(0) x(0)+\int_{-d(0)}^{0} x^{T}(s) e^{\lambda(t-s)} Q_{\sigma_{0}}(0) x(s) d s \\
& +\int_{-d}^{0} \int_{\theta}^{0} x^{T}(s) e^{\lambda(t-s)} Z_{\sigma_{0}}(0) x(s) d s d \theta \\
& \leq \alpha_{1} c_{1}+d e^{\lambda d} \alpha_{2} c_{1}+d^{2} e^{\lambda d} \alpha_{3} c_{1}
\end{aligned}
$$

where $\alpha_{1}=\max _{i \in M}\left\{\lambda_{\max }\left(P_{i}(0)\right)\right\}, \quad \alpha_{2}=\max _{i \in M}\left\{\lambda_{\max }\left(Q_{i}\right.\right.$ $(0))\}$, and $\alpha_{3}=\max _{i \in M}\left\{\lambda_{\max }\left(Z_{i}(0)\right)\right\}$. By (19) and (21),

$$
V_{\sigma(t)}\left(t, x_{t}\right) \leq \beta^{N_{\sigma}(0, t)} e^{\lambda t}\left(\alpha_{1} c_{1}+d e^{\lambda d} \alpha_{2} c_{1}+d^{2} e^{\lambda d} \alpha_{3} c_{1}\right) \text {. }
$$

According to (9),

$$
\begin{aligned}
V_{\sigma(t)}\left(t, x_{t}\right) & >x^{T}(t) P_{\sigma(t)}(t) x(t) \geq \lambda_{\min }\left(P_{\sigma(t)}(t)\right) x^{T}(t) x(t) \\
& \geq \lambda_{p} x^{T}(t) x(t) .
\end{aligned}
$$

Then, assuming that the total switching number of $\sigma(t)$ over $\left[0, T_{f}\right]$ is $N$. From (22) and (23),

$$
\begin{aligned}
x^{T}(t) x(t) & <\beta^{N_{\sigma}(0, t)} e^{\lambda t}\left(\alpha_{1} c_{1}+d e^{\lambda d} \alpha_{2} c_{1}+d^{2} e^{\lambda d} \alpha_{3} c_{1}\right) \lambda_{p}^{-1} \\
& =\beta^{N_{\sigma}(0, t)} e^{\lambda t} \eta_{1} \lambda_{p}^{-1} \\
& <\beta^{N} e^{\lambda T_{f}} \eta_{1} \lambda_{p}^{-1} .
\end{aligned}
$$

From the definition of ADT, $N=N_{\sigma}\left(0, T_{f}\right) \leq$ $N_{0}+T_{f} / \tau_{D}$. By inequality (7), we have

$$
\frac{T_{f} \ln \beta}{\ln c_{2}+\ln \lambda_{p}-\lambda T_{f}-\ln \eta_{1}+N_{0} \ln \beta}<\tau_{D} .
$$

It can be transformed into

$$
N_{0}+\frac{T_{f}}{\tau_{D}}<\frac{\ln c_{2}+\ln \lambda_{p}-\lambda T_{f}-\ln \eta_{1}}{\ln \beta},
$$

i.e.,

$$
N<\frac{\ln c_{2}+\ln \lambda_{p}-\lambda T_{f}-\ln \eta_{1}}{\ln \beta} .
$$

So, $N \ln \beta<\ln \left(c_{2} \lambda_{p} / e^{\lambda T_{f}} \eta_{1}\right)$, and

$$
\beta^{N} e^{\lambda T_{f}} \eta_{1} \lambda_{p}^{-1}<c_{2}
$$

Consequently, from (24), $x^{T}(t) x(t)<c_{2}$ for $t \in\left[0, T_{f}\right]$. System (1) is FTS with respect to $\left(c_{1}, c_{2}, T_{f}\right)$.

\section{Finite-Time Stability via State Feedback}

In this section, the LMIs conditions, introduced in Theorem 1, will be exploited to design a state feedback controller for the SLTV control system with time-varying delay. The resultant closed-loop system could be proved to be FTS. The problem is described concretely as follows. 
Given the time interval $\left[0, T_{f}\right]$, let us consider the SLTV control system:

$$
\begin{cases}\dot{x}(t)=A_{\sigma(t)}(t) x(t)+B_{\sigma(t)}(t) x(t-d(t))+G_{\sigma(t)} u(t), & t \in\left[0, T_{f}\right], \\ x(s)=\varphi(s), & \forall s \in[-\tau, 0]\end{cases}
$$

where $u(\cdot)$ represents the control input. The aim of this section is to find a memoryless, state feedback control law
$u(t)=K_{\sigma(t)}(t) x(t)$, such that the resultant closed-loop system

$$
\begin{cases}\dot{x}(t)=\left(A_{\sigma(t)}(t)+G_{\sigma(t)}(t) K_{\sigma(t)}(t)\right) x(t)+B_{\sigma(t)}(t) x(t-d(t)), & t \in\left[0, T_{f}\right], \\ x(s)=\varphi(s), & \forall s \in[-\tau, 0]\end{cases}
$$

is FTS with respect to $\left(c_{1}, c_{2}, T_{f}\right)$.

The following theorem states the sufficient conditions for the FTS of the closed-loop system (30) with respect to $\left(c_{1}, c_{2}, T_{f}\right)$.

Theorem 2. System (30) is FTS with respect to $\left(c_{1}, c_{2}, T_{f}\right)$ if there exist piecewise continuously differentiable positive definite matrix-functions $D_{i}(\cdot), H_{i}(\cdot)$, and $J_{i}(\cdot)$ and $a$ piecewise continuous matrix-function $L_{i}(\cdot)$ with appropriate dimensions such that the following LMIs hold, for $t \in\left[0, T_{f}\right]$,

$$
\left(\begin{array}{cc}
\Theta_{i}(t) & B_{i}(t) \\
B_{i}^{T}(t) & \Pi_{i}(t)
\end{array}\right)<0
$$

$\beta D_{i}(t) \geq D_{j}(t), H_{i}(t) \leq \beta H_{j}(t), J_{i}(t) \leq \beta J_{j}(t), \quad \forall i, j \in M$,

$$
\begin{gathered}
\dot{H}_{i}(t) \leq 0, \\
\dot{J}_{i}(t) \leq 0,
\end{gathered}
$$

and the ADT satisfies

$$
\tau_{D}>\frac{T_{f} \ln \beta}{\ln c_{2}+\ln \gamma_{p}-\lambda T_{f}-\ln \delta_{1}+N_{0} \ln \beta},
$$

where

$$
\begin{aligned}
\Theta_{i}(t)= & D_{i}(t) A_{i}^{T}(t)-\dot{D}_{i}(t)+A_{i}(t) D_{i}(t)+H_{i}(t) \\
& +d J_{i}(t)-\lambda D_{i}(t) \\
& +G_{i}(t) L_{i}(t)+L_{i}^{T}(t) G_{i}^{T}(t), \\
\Pi_{i}(t)= & -e^{\lambda d} H_{i}(t)(1-\rho), \\
\mu_{1}= & \max _{i \in M}\left\{\lambda_{\max }\left(D_{i}^{-1}(0)\right)\right\}, \\
\mu_{2}= & \max _{i \in M}\left\{\lambda_{\max }\left(D_{i}^{-1}(0) H_{i}(0) D_{i}^{-1}(0)\right)\right\}, \\
\mu_{3}= & \max _{i \in M}\left\{\lambda_{\max }\left(D_{i}^{-1}(0) J_{i}(0) D_{i}^{-1}(0)\right)\right\}, \\
\gamma_{p}= & \min _{i \in M}\left(\lambda_{\min }\left(D_{i}^{-1}(t)\right)\right),
\end{aligned}
$$

and the state feedback controller gain $K_{i}(t)=L_{i}(t) D_{i}^{-1}(t), \forall i \in M$.

Proof. To prove the finite time stability of system (30), we only need to prove that system (30) satisfies all the conditions of Theorem 1. Concretely, we only need to prove conditions (3)-(6) are equivalent to (31)-(34), separately.

Now, we prove the equivalence of (3) and (31). For system (30), from (3), we have

$$
\left(\begin{array}{cc}
\Phi_{i}(t) & P_{i}(t) B_{i}(t) \\
B_{i}^{T}(t) P_{i}(t) & -e^{\lambda d} Q_{i}(t)(1-\rho)
\end{array}\right)<0,
$$

where $\Phi_{i}(t)=\left(A_{\sigma(t)}(t)+G_{\sigma(t)}(t) K_{\sigma} \quad(t)(t)\right)^{T} P_{i}(t)+\dot{P}_{i}$ $(t)+P_{i}(t)\left(A_{\sigma}(t)(t)+G_{\sigma(t)}(t) K_{\sigma(t)}(t)\right)+Q_{i}(t)+d Z_{i}(t)-$ $\lambda P_{i}(t)$. Let $D_{i}(t)=P_{i}^{-1}(t)$; we pre- and postmultiply (37) by the block tridiagonal matrix $\left(D_{i}(t), I\right)$, and we obtain

$$
\left(\begin{array}{cc}
\Upsilon_{i}(t) & B_{i}(t) \\
B_{i}^{T}(t) & \Psi_{i}(t)
\end{array}\right)<0,
$$

where

$$
\begin{aligned}
\Upsilon_{i}(t)= & D_{i}(t) A_{i}^{T}(t)+D_{i}(t) G_{i}^{T}(t) K_{i}^{T}(t)-\dot{D}_{i}(t) \\
& +A_{i}(t) D_{i}(t) \\
& +G_{i}(t) K_{i}(t) D_{i}(t)+D_{i}(t) Q_{i}(t) D_{i}(t) \\
& +d D_{i}(t) Z_{i}(t) D_{i}(t)-\lambda D_{i}(t) \\
\Psi_{i}(t)= & -e^{\lambda d} D_{i}(t) Q_{i}(t)(t) D_{i}(t)(1-\rho) .
\end{aligned}
$$

Then, from $K_{i}(t)=L_{i}(t) D_{i}^{-1}(t), H_{i}(t)=D_{i}(t) Q_{i}(t) D_{i}$ $(t)$, and $J_{i}(t)=D_{i}(t) Z_{i}(t) D_{i}(t)$, it can be obtained that $\Theta_{i}(t)=\Upsilon_{i}(t)$ and $\Pi_{i}(t)=\Psi_{i}(t)$, and further, (31) and (3) are equivalent. In the same way, equivalence between (4) and (32) is straightforward. Similarly, (5) and (33) are equivalent, (6) and (34) are equivalent. Therefore, from Theorem 1, the closed-loop system (30) is FTS. 
Remark 2. Here, it is assumed that there is no delay between the controllers and the subsystems, i.e., the control act on the subsystems without delay.

\section{Numerical Example}

In this section, a numerical example will be given to illustrate the validity of our results.

Example 1. Consider the following SLTV system:

$$
\left\{\begin{array}{rlrl}
\dot{x}(t)= & A_{\sigma(t)}(t) x(t)+B_{\sigma(t)}(t) x(t-d(t)) & \\
& +G_{\sigma(t)} u(t), & & t \in\left[0, T_{f}\right], \\
x(s)= & \varphi(s), & & \forall s \in[-\tau, 0),
\end{array}\right.
$$

where $\sigma(t) \in M=\{1,2\}, d(t)=0.0001 t$,

$$
\begin{aligned}
& A_{1}=\left(\begin{array}{cc}
0.01 t & 0.01 t \\
0.1 \cos (t) & 0.1
\end{array}\right), \\
& B_{1}=\left(\begin{array}{cc}
0.01 & -0.01 t \\
0.1 \sin (t) & 0.01
\end{array}\right), \\
& G_{1}=\left(\begin{array}{c}
1 \\
4
\end{array}\right), \\
& A_{2}=\left(\begin{array}{cc}
0.01 & -0.01 t \\
0.1 \sin (t) & 0.01
\end{array}\right), \\
& B_{2}=\left(\begin{array}{cc}
0.01 t & 0.01 t \\
0.1 \cos (t) & 0.1
\end{array}\right), \\
& G_{2}=\left(\begin{array}{l}
4 \\
1
\end{array}\right) .
\end{aligned}
$$

Let $c_{1}=1, c_{2}=8$, and $T_{f}=10$ be given. The switching law of system (40) is shown in Figure 1, and it satisfies the ADT parameters $\tau_{D}=1.25$ and $N_{0}=1$. With this switching law, Figure 2 shows the system state of system (40) without control and with initial state $(-0.10 .5)^{\mathrm{T}}$. From Figure 2, it can be seen that the system state is unbounded and is not FTS.

From inequality (7), we let $\lambda=0.1$ and $\beta=1.05$. Applying Theorem 2, we design a finite time stable state feedback controller $u(t)=K_{\sigma(t)}(t) x(t)$. Figure 3 shows the time traces for the two elements of state feedback controller gain $K_{\sigma(t)}(t)$. It can be seen from Figure 4 that, with the state feedback controller, the system state with initial state $(-0.10 .5)^{\mathrm{T}}$ satisfies $x^{\mathrm{T}}(t) x(t)<c_{2}=8$ over $[0,10]$.

Remark 3. Since $P(t), Q(t)$, and $Z(t)$ are continuously time varying, LMIs cannot be solved directly. In order to

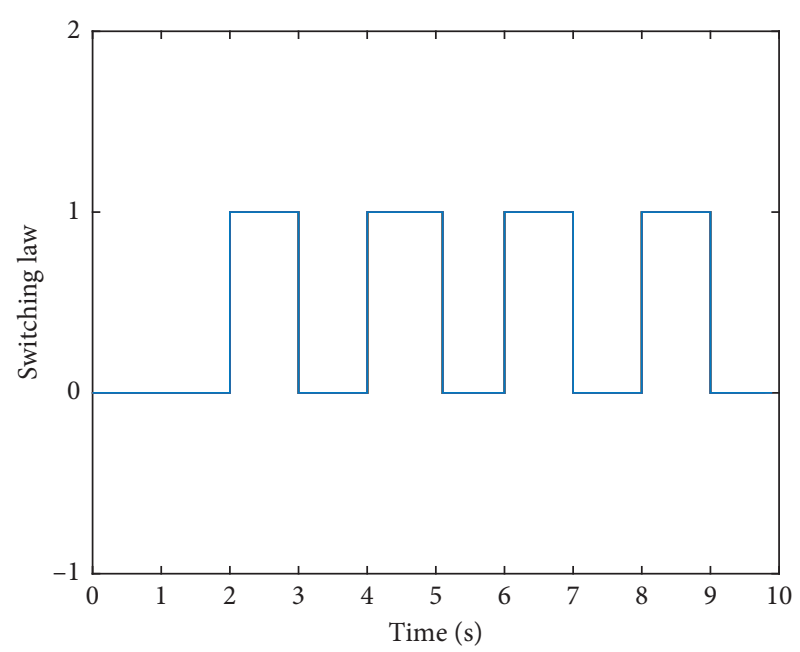

FIgURE 1: Switching law.

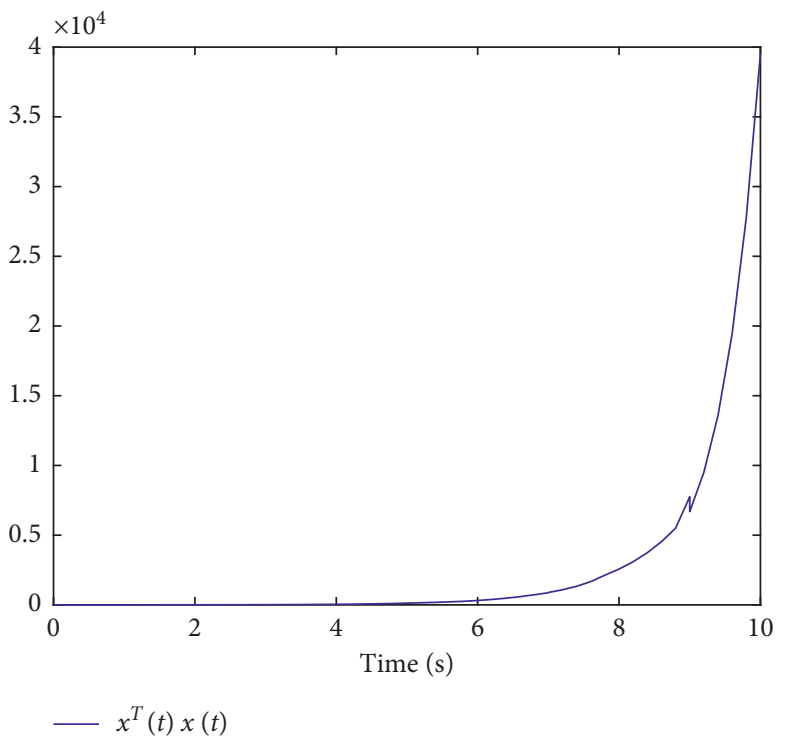

Figure 2: State of system (40) with $u \equiv 0$.

get a computationally tractable problem, we discretize the time interval into equally spaced subintervals. If the length of the subintervals is sufficient small, the continuous functions can be approximated by piecewise functions. Then, the LMIs can be solved. This approximating method can also be found in [26, 27]. Concretely, discretize $\left[0, T_{f}\right]$ into equally spaced time instances $t_{i}(i=$ $0,1,2, \ldots, N)$ with $t_{0}=0, t_{N}=T_{f}$, and $t_{k}-t_{k-1} \triangleq$ $\varepsilon=\left(T_{f} / N\right)$. Thus, on the time interval $\left(t_{k-1}, t_{k}\right]$ with small enough $\varepsilon$, the time-varying matrix-valued functions $P(t), Q(t)$, and $Z(t)$ can be denoted as $P(t)=P\left(t_{k}\right), Q(t)=Q\left(t_{k}\right)$, and $Z(t)=Z\left(t_{k}\right), \quad$ for $t \in\left(t_{k-1}, t_{k}\right]$. Then, $\dot{P}(t)$ can be denoted as $\left(P\left(t_{k}\right)-P\left(t_{k-1}\right) / \varepsilon\right)$ with sufficiently small $\varepsilon$. Similarly, $\dot{Q}(t)$ and $\dot{Z}(t)$ can be replaced by $\left(Q\left(t_{k}\right)-Q\left(t_{k-1}\right) / \varepsilon\right)$ and $\left(Z\left(t_{k}\right)-Z\left(t_{k-1}\right) / \varepsilon\right)$. 


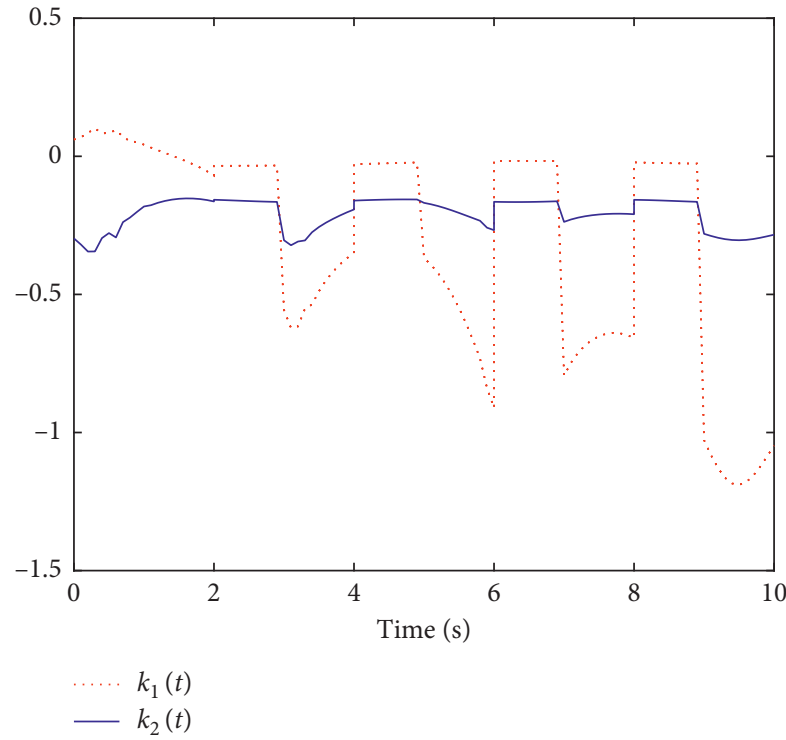

Figure 3: Time traces of the elements in state feedback controller gain $K_{i}(t)=\left(k_{1}(t), k_{2}(t)\right)$.

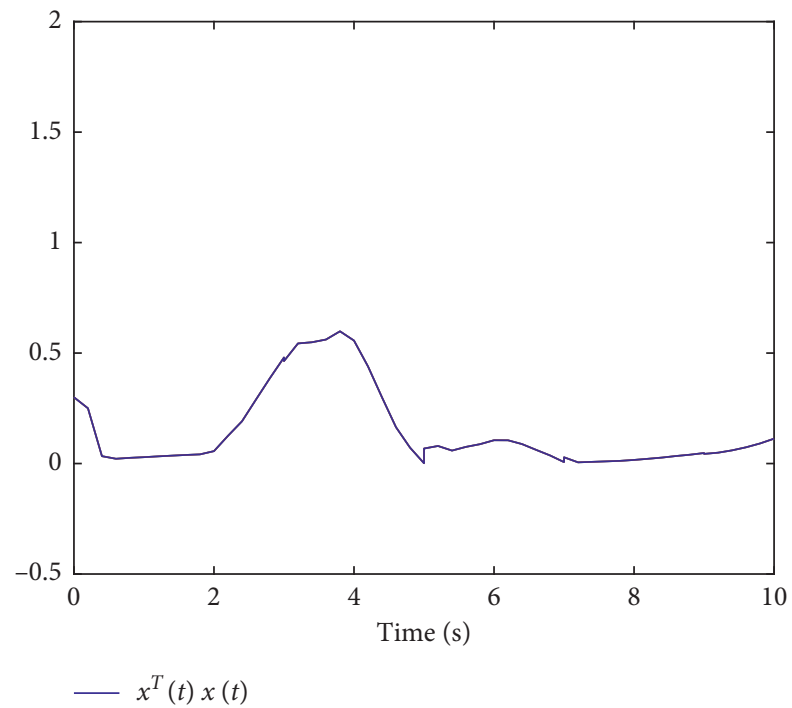

FIGURE 4: State of the resultant closed-loop system of system (40).

\section{Conclusions}

This paper focuses on the FTS analysis of SLTV systems with time-varying delays. For this kind of systems, based on the Lyapunov-Krasovskii functional technique and ADT method, a sufficient criterion on FTS is obtained. Based on this criterion, using the LMI method, a state feedback controller is designed such that the system is FTS. Finally, a numerical example illustrates the validity of the obtained results. The innovation mainly lies in the studying of FTS for the switched system on the basis of the LTV system, which has become more and more important now. And, a timevarying state feedback controller is designed. Future efforts will be devoted to obtain less conservative criteria for the finite-time stability of linear time-varying system and extend the FTS theory to the neural network context.

\section{Data Availability}

No data were used to support this study. The authors only used MATLAB for simulation. Therefore, simulation programming can be obtained from the corresponding author upon request.

\section{Conflicts of Interest}

The authors declare that they have no conflicts of interest.

\section{Acknowledgments}

This work was supported by the Shandong Provincial Natural Science Foundation, China (no. ZR2017JL028).

\section{References}

[1] D. Liberzon, Switching in Systems and Contro, Springer, Boston, MA, USA, 2003.

[2] A. Jadbabaie, J. Jie Lin, and A. S. Morse, "Coordination of groups of mobile autonomous agents using nearest neighbor rules," IEEE Transactions on Automatic Control, vol. 48, no. 6, pp. 988-1001, 2003.

[3] X. M. Dong, Y. Zhou, Z. Ren, and Y. S. Zong, “Time-varying formation tracking for second-order multi-agent systems subjected to switching topologies with application to quadrotor formation flying," IEEE Transactions on Industrial Electronics, vol. 64, no. 6, pp. 5014-5024, 2016.

[4] D. Zhang, P. Shi, W. A. Zhang, and L. Yu, "Energy-efficient distributed filtering in sensor networks: a unified switched system approach," IEEE Transactions on Cybernetics, vol. 47, no. 7, pp. 1618-1629, 2016.

[5] D. Zhang, P. Shi, and Q.-G. Wang, "Energy-efficient distributed control of large-scale systems: a switched system approach," International Journal of Robust and Nonlinear Control, vol. 26, no. 14, pp. 3101-3117, 2016.

[6] W. Xiang, J. Xiao, and M. N. Iqbal, "Asymptotic stability, $\ell 2$ gain, boundness analysis, and control synthesis for switched systems: a switching frequency approach," International Journal of Adaptive Control and Signal Processing, vol. 26, no. 4, pp. 350-373, 2012.

[7] X. Wang and J. Zhao, "Autonomous switched control of load shifting robot manipulators," IEEE Transactions on Industrial Electronics, vol. 64, no. 9, pp. 7161-7170, 2017.

[8] L. Weiss and E. Infante, "Finite time stability under perturbing forces and on product spaces," IEEE Transactions on Automatic Control, vol. 12, no. 1, pp. 54-59, 1967.

[9] A. N. Michel and S. H. Wu, "Stability of discrete systems over a finite interval of timet," International Journal of Control, vol. 9, no. 6, pp. 679-693, 1969.

[10] S. Mastellone, C. T. Abdallah, and P. Dorato, "Stability and finite-time stability analysis of discrete-time nonlinear networked control systems," in Proceedings of the 2015 IEEE/ American Control Conference (ACC), pp. 1239-1244, IEEE, Chicago, IL, USA, July 2015.

[11] L. J. Wang, L. Cai, and X. Z. Liu, "Practical stability and bounds of heterogeneous AIMD/RED system with time delay," in Proceedings of the 2008 IEEE International Conference 
on Communications (ICC), pp. 5558-5563, IEEE, Beijing, China, May 2008.

[12] G. Zong, W. Qi, and H. R. Karimi, " $\mathscr{L}_{1}$ control of positive semimarkov jump systems with state delay," IEEE Transactions on Systems, Man, and Cybernetics: Systems, pp. 1-10, 2020.

[13] G. Zong and H. Ren, "Guaranteed cost finite-time control for semi-Markov jump systems with event-triggered scheme and quantization input," International Journal of Robust and Nonlinear Control, vol. 29, no. 15, pp. 5251-5273, 2019.

[14] X. Wang, G. Zong, and H. Sun, "Asynchronous finite-time dynamic output feedback control for switched time-delay systems with non-linear disturbances," IET Control Theory \& Applications, vol. 10, no. 10, pp. 1142-1150, 2016.

[15] H. Ren, G. Zong, and T. Li, "Event-triggered finite-time control for networked switched linear systems with asynchronous switching," IEEE Transactions on Systems, Man, and Cybernetics: Systems, vol. 48, no. 11, pp. 1874-1884, 2018.

[16] H. L. Ren, G. D. Zong, and H. R. Karimi, "Asynchronous finite-time filtering of networked switched systems and its application: an event-driven method," IEEE Transactions on Circuits and Systems I: Regular Papers, vol. 66, no. 1, pp. 391-402, 2018.

[17] T. Yu, Y. Zhong, T. Chen, and C. Chen, "Finite-time stabilization of uncertain switched positive linear systems with time-varying delays," Discrete Dynamics in Nature and Society, vol. 2015, Article ID 954782, 9 pages, 2015.

[18] H. Y. Li, J. Y. Yu, and C. Hilton, "Adaptive sliding-mode control for nonlinear active suspension vehicle systems using TCS fuzzy approach," IEEE Transactions on Industrial Electronics, vol. 60, no. 8, pp. 3328-3338, 2012.

[19] Y. K. Zhong and T. F. Chen, "Finite-time boundedness analysis for a class of switched linear systems with timevarying delay," Abstract and Applied Analysis, vol. 2014, Article ID 982414, , 2014.

[20] B. A. Francis and T. T. Georgiou, "Stability theory for linear time-invariant plants with periodic digital controllers," IEEE Transactions on Automatic Control, vol. 33, no. 9, pp. 820832, 1988.

[21] K. S. Tsakalis and P. A. Ioannou, Linear Time-Varying Systems: Control and Adaptation, Prentice-Hall, Upper Saddle River, NJ, USA, 1993.

[22] N. T. K. Son, N. P. Dong, L. H. Son, M. Abdel-Basset, G. Manogaran, and H. V. Long, "On the stabilizability for a class of linear time-invariant systems under uncertainty," Circuits, Systems, and Signal Processing, vol. 39, no. 2, pp. 919-960, 2020.

[23] M.-Y. Wu, I. M. Horowitz, and J. C. Dennison, "On solution, stability and transformation of linear time-varying systems," International Journal of Control, vol. 22, no. 2, pp. 169-180, 1975.

[24] H. Jing, X. Li, and H. Karimi, "Output-feedback based $H_{\infty}$ control for active suspension systems with control delay," IEEE Transactions on Industrial Electronics, vol. 64, no. 4, pp. 436-446, 2014.

[25] Z. C. Li, H. C. Yan, H. Zhang, X. S. Zhan, and C. Z. Huang, "Improved inequality-based functions approach for stability analysis of time delay system," Automatica, vol. 108, Article ID 108416, 2019.

[26] F. Amato, R. Ambrosino, C. Cosentino, and G. De Tommasi, "Input-output finite time stabilization of linear systems," Automatica, vol. 46, no. 9, pp. 1558-1562, 2010.

[27] F. Amato, M. Ariola, and C. Cosentino, "Finite-time stability of linear time-varying systems: analysis and controller design," IEEE Transactions on Automatic Control, vol. 55, no. 4, pp. 1003-1008, 2010. 